\title{
APORTES DEL FEMINISMO A LA LUCHA SOCIOAMBIENTAL
}

\author{
Ana Mariel Weinstock \\ Universidad Nacional de San Martin (UNSAM)
}

\begin{abstract}
Resumen: A partir de mi tesis de maestría, comencé a interesarme por los cruces entre el pensamiento ambiental y las teorizaciones feministas. Las I y II Jornadas de feminismos pos y descoloniales en Buenos Aires (2010 y 2012) organizadas por el Programa "Poscolonialidad, pensamiento fronterizo y transfronterizo en los estudios feministas" (IDAES-UNSAM) resultaron un marco ideal para estas discusiones. Este ensayo es un primer intento por abordar las actuales luchas socioambientales por la tierra, el territorio y el modelo de desarrollo, en la provincia de Chubut, desde la teoría feminista.

Palabras clave: mujer; naturaleza; opresión; género; cosmovisión originaria.
\end{abstract}

\section{Introdución. La necesaria y peligrosa proximidad mujer- naturaleza}

Desde sus inicios, las teorizaciones feministas han problematizado la relación naturaleza-cultura en relación a las diferencias de sexos. Se han interrogado acerca de los condicionantes biológicos y socioculturales y así han aportado a un debate mayor sobre qué es lo determinante en el comportamiento humano: ¿lo innato o lo adquirido? ${ }^{2}$ Específicamente, nos interesa la denuncia feminista sobre la asociación desvalorizante que establece el patriarcado entre la mujer y la naturaleza. A partir de aquí, quedó teóricamente aceptada la idea de que el control del cuerpo femenino y de los territorios naturales responden a un mismo patrón de dominio. Sin duda, un aporte valioso, porque permite conceptualizar la opresión desde un punto de vista sistémico, holístico, poscolonial e interseccional: desde "el sistema moderno-colonial de género", según María Lugones.

Adoptando el enfoque de la colonialidad de Aníbal Quijano, Lugones afirma que la categoría "género" es tan central e indispensable como la categoría "raza" para la vigencia del patrón colonial del poder y del saber. Ella explica que no es necesario que las relaciones

Copyright @ 2014 by Revista Estudos Feministas.

1 Realizado en el marco de la Investigación Plurianual PIP -CONICET: "Violencia en mujeres subalternas. Representaciones de I desigualdad de género en políticas culturales" (IDAES/UNSAM).

${ }^{2}$ Marta LAMAS, 1986. 
sociales estén organizadas en términos de género, ni siquiera aquellas relaciones que se consideren sexuales:

Tanto el dimorfismo biológico, el heterosexualismo, como el patriarcado son característicos de lo que llamo el lado claro/visible de la organización colonial/moderna del género. El dimorfismo biológico, la dicotomía hombre/mujer, el heterosexualismo, y el patriarcado están inscriptos con mayúsculas, y hegemónicamente en el significado mismo del género.

El lado oscuro/oculto de la organización colonial/moderna del género se basa en la construcción de una categoría homogénea de mujer, eurocentrada y válida universalmente, que se corresponde con las características de mujer blanca, burguesa, clase media e intelectual que reivindica el feminismo hegemónico. ${ }^{4}$ Para Lugones, es fundamental visualizar este lado oculto, porque nos "permitiría desenmascarar esa colaboración cómplice, y nos convocaría a rechazarla en las múltiples formas a través de las cuales se expresa al mismo tiempo que reanudamos nuestro compromiso con la integridad comunal en una dirección liberatoria". ${ }^{5}$

Sin embargo, conviene revisar cuál es el fundamento teórico de asimilar la soberanía patriarcal sobre el cuerpo femenino y sobre la naturaleza, para no caer en afirmaciones cristalizadas y naturalizaciones acríticas que terminan conspirando contra un pensamiento pretendidamente emancipatorio que las generó.

En efecto, pensadoras enroladas dentro del ecofeminismo señalaron que existen importantes paralelos históricos, culturales y simbólicos entre la opresión y explotación de las mujeres y de la naturaleza. De hecho, en los discursos patriarcales, la dicotomía mujer/ hombre corresponde al de naturaleza/civilización, emoción/razón o incluso tradición/ modernidad, desvalorizando la primera categoría del binomio. ${ }^{6}$ Se trataba así, de deconstruir una dicotomía donde la mujer quedaba subsumida en el primer término. Al igual que en el emblemático, potente y revelador "lo personal es político", (la dicotomía es este caso: sujetx/sociedad), la negación del carácter escindido de los términos permite visualizar las relaciones de poder ocultas bajo una mirada disociada.

Ahora bien, asociar mujer y naturaleza como oprimidas puede aparejar dos significaciones distintas. Por un lado, como mencionamos, la mirada sistémica y la deconstrucción de la dicotomía sujeta/sociedad. Por otro lado, un nuevo modo de esencialismo tanto natural como cultural. "Muchas veces en los discursos sobre el buen vivir, en un esencialismo cultural, se termina atribuyendo a las mujeres indígenas el papel de guardianas de la cultura, vistiendo traje tradicional, mientras que los hombres occidentalizan su aspecto para migrar a las ciudades. Esto sin que paralelamente se asuma el compromiso político de criticar todo aquello que al interior de las culturas produce desigualdad de género". ${ }^{7}$ Es decir, esta ligazón mujer-naturaleza puede conducir a dos perspectivas muy distintas: 1) igualar a la mujer y a la naturaleza en su condición de objetos oprimidas dentro de la actual matriz de poder; y 2) considerar a la mujer el sujeto único de la liberación por alguna característica naturalmente innata.

${ }^{3}$ María LUGONES, 2008, p. 78

4 "[...] es parte de su historia, que en el Occidente, sólo las mujeres burguesas blancas han sido contadas como mujeres. Las hembras excluidas por y en esa descripción no eran solamente sus subordinadas sino también eran vistas y tratadas como animales, en un sentido más profundo que el de la identificación de las mujeres blancas con la naturaleza, con los niños, y con los animales pequeños. Las hembras no-blancas eran consideradas animales en el sentido profundo de ser seres "sin género", marcadas sexualmente como hembras, pero sin las características de la femineidad (LUGONES, 2008, p. 94).

5 LUGONES, 2008, p. 99.

${ }^{6}$ Margarita AGUINAGA, 2012

7 AGUINAGA, Miriam LANG, Dunia MOKRANI y Alejandra SANTILLANA, 2012, p. 68 
En este sentido, resulta útil reconocer y diferenciar tendencias teóricas que existen dentro del ecofeminismo. Una corriente considera a la mujer más cerca de la naturaleza que los varones, debido a una supuesta esencia femenina. En ella se depositan las esperanzas de la humanidad y la conservación de la naturaleza porque "naturalmente" es más proclive a la defensa de los seres vivos y a una ética del cuidado. Sin embargo, otra corriente, entre quienes se encuentran Vandana Shiva, María Mies y Bina Agarwal, rechaza este esencialismo y ubica el origen de una mayor compatibilidad de las mujeres con la naturaleza en la construcción social e histórica del género, específica en cada cultura. La conciencia ecológica de género para ellas nace de las divisiones de trabajo y roles sociales concretos establecidos en los sistemas históricos de género y clase, y en las relaciones de poder político y económico asociado con ellos - por ejemplo, cuando las mujeres asumen en la repartición de tareas familiares y comunitarias la búsqueda de leña y/o agua, o el cuidado de los huertos. ${ }^{8}$

Inscripto en este debate, en la Conferencia de los Pueblos sobre Cambio Climático (2010), el feminismo comunitario afirmaba:

\begin{abstract}
Denunciamos que la comprensión de Pachamama como sinónimo de Madre Tierra es reduccionista y machista, que hace referencia solamente a la fertilidad para tener a las mujeres y a la Pachamama a su arbitrio patriarcal. 'Madre Tierra', es un concepto utilizado hace varios años y que se intenta consolidar en esta Conferencia de los pueblos sobre Cambio Climático con la intención de reducir a la Pachamama -así como nos reducen a las mujeres- a su función de útero productor y reproductor al servicio del patriarcado. Entienden a la Pachamama como algo que puede ser dominada y manipulada al servicio del "desarrollo" y del consumo- y no la conciben como el cosmos del cual la humanidad sólo es una pequeña parte. El cosmos, No Es, el "Padre Cosmos". El cosmos es parte de la Pachamama. No aceptamos que "casen", que obliguen al matrimonio a la Pachamama. [...] Cuando hablan del "padre Cosmos" intentan minimizar y subordinar a la Pachamama a un Jefe de Familia masculino y heterosexual. Pero, ella, la Pachamama, es un todo y no nos pertenece. Nosotras y nosotros somos de ella [...].
\end{abstract}

\title{
¿Es la mujer para el sexo y el género, lo que el extractivismo para la naturaleza y la sociedad?
}

Todas estas disquisiciones nos remiten a la pregunta de Verena Stolcke:10 "¿Es el sexo para el género, lo que la raza para la etnicidad... y la naturaleza para la sociedad?", que aquí podríamos hacer jugar en paralelo construyendo una nueva pregunta: "¿Es la mujer para el sexo y el género, lo que el extractivismo para la naturaleza y la sociedad?"."1

Particularmente, me interesa esta pregunta porque estoy involucrada personalmente en la lucha contra el modelo extractivo de megaminería a cielo abierto que afecta mi región y que inevitablemente nos lleva a cuestionarnos cuál es nuestra relación con la naturaleza en la era de la hipertecnologización. En un momento donde el desarrollo de la ingeniería y de la creatividad parecía desafiar y destruir cualquier condicionamiento o límite, aparece la pregunta lisa, llana y básica sobre qué sentido tienen todos estos "avances", y cuánto aportan a la felicidad al ser humano. Cuanto más la humanidad quiso desvincularse de la naturaleza, más dependiente se descubrió. Centralmente, me preocupa un riesgo que observo en la militancia cotidiana: la posibilidad de que cierto

${ }^{8}$ Susan PAULSON, 1998, citado por AGUINAGA et al., 2011.

${ }^{9}$ Disponible en: </http://old.kaosenlared.net/noticia/pronunciamiento-feminismo-comunitario-latinoamericanoconferencia-pueb>. Acceso en: 20 enero de 2014.

${ }^{10}$ Verena STOLCKE, 2000

1 Para un rastreo histórico de este debate, ver Sherry ORTNER, 1974. 
nivel de dogmatismo en la resistencia derive en un esencialismo de clase (los pobres son revolucionarios en sí mismos); de raza (las comunidades mapuche "deben" encabezar esta lucha, más allá de su experiencia colectiva); de género (por cosmovisión, las mujeres mapuche no padecen relaciones de subordinación dentro de su comunidad).

En el mencionado artículo, Stolcke revisa la homología entre naturaleza y sexo al igual que la persistente (no por antigua, menos efectiva) dicotomía naturaleza-cultura' ${ }^{2}$; sexo-género y raza-etnicidad en el pensamiento académico. Realiza un análisis histórico de dichas categorías, postulando la naturalización las diferencias sociales con fines de dominación política-económica y la estrecha relación entre racismo y sexismo. Y aunque persiste la dificultad para saber hasta qué punto sexo y género, o naturaleza y cultura se intersectan, los términos en que hoy se plantea dicha relación han cambiado. Stolcke plantea que si bien se ha intensificado la polarización epistemológica entre constructivistas radicales (para quienes todo conocimiento y toda ciencia y sus criaturas como la 'naturaleza' o el 'sexo' son invariablemente el resultado de maniobras de poder) y las empiristas críticas que, en cambio, aspiran a desmitificar un mundo que después de todo es 'real', en los años noventa también se han podido vislumbrar diversas iniciativas para trascender tanto los constructivismos como los empirismos universalistas, y lograr una nueva visión holística y dinámica de la naturaleza en relación con la sociedad y la cultura.

Reconocer que la concepción occidental de la 'naturaleza' también es irrecuperablemente social e histórica, deja pendiente, no obstante, dos cuestiones importantes. Por un lado, y a pesar de sus pretensiones contrarias, el enfoque constructivista no está él mismo ajeno al dualismo cartesiano en la medida en que sólo puede sostenerse que esto o aquello es socialmente construido al suponer que hay un ámbito que es claramente distinto y distinguible de la 'cultura', a saber, la 'naturaleza' que no lo es. Y, por otro lado, se plantea el dilema de cómo abordar desde una perspectiva historicista la materialidad y solidez de fenómenos y transformaciones bio-fisiológicos tales como dimorfismo sexual cuando, por ejemplo, la clonación prescinde de él en la procreación. ${ }^{13}$

La nominación no es un problema superficial, ya que con la acción teórica de categorizar, la ciencia o cualquier análisis reflexivo construye realidades, y, de alguna manera, también construye "su" "objeto de estudio".

\begin{abstract}
La interseccionalidad ${ }^{14}$ revela lo que no se ve cuando categorías como género y raza se conceptualizan como separadas unas de otra. La denominación categorial construye lo que nomina. Las feministas de color nos hemos movido conceptualmente hacia un análisis que enfatiza la intersección de las categorías raza y género porque las categorías invisibilizan a quienes somos dominadas y victimizadas bajo la categoría «mujer» y bajo las categorías raciales "Black", "hispanic", "Asian", "Native American", "Chicana" a la vez, es decir a las mujeres de color [...] En la intersección entre «mujer» y «negro» hay una ausencia donde debería estar la mujer negra precisamente porque ni «mujer» ni «negro» la incluyen. La intersección nos muestra un vacío. Por eso, una vez que la interseccionalidad nos muestra lo que se pierde, nos queda por delante la tarea de reconceptualizar la lógica de la intersección para, de ese modo, evitar la separabilidad de las categorías dadas y el pensamiento categorial. Sólo al percibir género y raza como entretramados o fusionados indisolublemente, podemos realmente ver a las mujeres de color. ${ }^{15}$
\end{abstract}

\footnotetext{
12 "Culturaleza" es el término que utilizo en mi tesis de maestría para conceptualizar cómo percibe y siente el territorio el movimiento socioambiental (Ana Mariel WEINSTOCK, 2012, p. 23).

${ }^{13}$ STOLCKE, 2000, p. 54.

${ }^{14}$ Con este concepto, Lugones propone concebir como simultáneamente interconectadas las categorías de clase, raza y género.

${ }^{15}$ LUGONES, 2008, p. 81-82.
} 
Así como Quijano nos muestra que el rasgo fenotípico no es una diferencia biológica que pueda explicar la categoría raza; tampoco el rasgo sexual es una diferencia biológica que pueda explicar la categoría género. ${ }^{16}$ Las feministas han teorizado sobre la diferenciación entre sexo y género, y con sus análisis, pusieron de manifiesto las dificultades de dicha diferenciación. Hoy por hoy continuamos necesitando "desentrañar las diferencias que son inevitables de aquellas que son elegidas y de aquellas otras que son simplemente impuestas". ${ }^{17}$ En palabras de Boaventura de Sousa Santos, ${ }^{18}$ debemos defender el derecho a la diferencia, toda vez que la igualdad nos descaracterice, al mismo tiempo que defender el derecho a la igualdad, toda vez que la diferencia nos inferiorice.

\section{Diferencias e igualdades en las comunidades originarias}

La relación entre mujer y naturaleza siempre resultó más fácil de observar en el caso de mujeres indígenas debido a la cosmovisión en las que ellas están inscriptas. En ella, la tierra no pertenece a los seres humanos, sino que son los humanos los que pertenecen a la tierra.

Así lo muestra la historia de Gladys Villar Ñanco, quien junto a su marido fundaron la Comunidad Mapuche Tehuelche Pu Fotum Mapu ("Hijos de la tierra") en la ciudad patagónica de Puerto Madryn. Ella fue la guardiana del predio que obtuvieron en las afueras de la urbe, que cuidó y protegió como si fuera un hijo o hija más.

La fuerte conexión entre mujer y naturaleza también se puede apreciar en palabras de Paula Castia, de la comunidad de Pu Kona Mapu ("Guerreros de la tierra"), quien organizó el Primer Encuentro de Mujeres Originarias en Puerto Madryn: "[...] la mayoría de mujeres que estuvieron en el encuentro fueron víctimas de abuso. De abuso contra su cuerpo. De abuso psicológico. O sea: lo mismo que le está pasando a la tierra, nos está pasando a nosotras". ${ }^{19}$ Según Rita Segato, distintas evidencias históricas y relatos etnográficos muestran de forma incontestable la existencia de nomenclaturas de género en las sociedades tribales y afroamericanas. Es decir, en las culturas originarias podemos reconocer estructuras de diferencia semejantes a lo que llamamos relaciones de género en la modernidad, con jerarquías claras de prestigio entre la masculinidad y la feminidad, representados por figuras que pueden ser entendidas como hombres y mujeres. Pero si bien el género existe, lo hace de una forma diferente que en la modernidad. Y cuando esa colonial/modernidad se le aproxima al género de la aldea, lo modifica peligrosamente, interviniendo, capturando y reorganizando desde dentro de la comunidad, manteniendo la apariencia de continuidad, pero transformando los sentidos, al introducir un orden ahora regido por la conquista..$^{20}$

En sentido similar, Allen razona que muchas comunidades tribales de Nativos Americanos eran matriarcales, reconocían positivamente tanto a la homosexualidad como al "tercer" género, y entendían al género en términos igualitarios, no en los términos de subordinación que el capitalismo eurocentrado les terminó por imponer. ${ }^{21}$ Lugones cita a Oyéronké Oyewùmi ${ }^{22}$ para preguntarse si patriarcado es una categoría transcultural válida y así habilitar la sentencia

\footnotetext{
16 "La naturalización de las diferencias sexuales es otro producto del uso moderno de la ciencia que Quijano subraya para el caso de la 'raza'. Es importante notar que la gente intersexual no es corregida ni normalizada por todas las diferentes tradiciones. Por eso, como lo hacemos con otras suposiciones, es importante preguntarse de qué forma el diformismo sexual sirvió, y sirve, a la explotación/dominación capitalista global eurocentrada" (LUGONES, 2008, p. 86)

17 STOLCKE, 2000 , p. 54.

${ }^{18}$ Boaventura de Sousa SANTOS, 2003

${ }^{19}$ Entrevista a Paula Castia, marzo de 2012.

20 Rita SEGATO, 2011 , p. 30.

${ }^{21}$ LUGONES, 2008, p. 86

22 Oyéronké OYEWÙMI 1997 apud LUGONES 1998, p. 90.
} 
de que "el género no era un principio organizador en la sociedad Yoruba antes de la colonización Occidenta". Aquí, patriarcado no está concebido como el opuesto a matriarcado, sino para resaltar que no había un sistema de género institucionalizado.

Incluso, Oyewùmi nos indica que el género ha adquirido importancia en los estudios Yoruba no como un artefacto de la vida Yoruba sino porque ésta, tanto en lo que respecta a su pasado como su presente, ha sido traducida al Inglés para encajar en el patrón Occidental de separación entre cuerpo y razón [...]. Asumir que la sociedad Yoruba incluía el género como un principio de organización social es otro caso de "dominación Occidental sobre la documentación e interpretación del mundo; una dominación que es facilitada por el dominio material que Occidente ejerce sobre el globo" [...] Oyewùmi afirma que los/as investigadores siempre encuentran el género cuando lo están buscando.

Quijano explicó claramente que el mito de la colonialidad reside en afirmar que Europa pre-existió al patrón capitalista de poder y que, por lo tanto, constituía el momento más avanzado en el curso continuo, unidireccional, y lineal de las especies. Según esta creencia, la humanidad se diferenció en dos grupos: superior e inferior, racional e irracional, primitivo y civilizado, tradicional y moderno. Así, los humanos no europeos llegaron a ser míticamente concebidos ya no como dominados a través de la conquista, ni como inferiores en términos de riqueza o poder político, sino como etapa anterior en la historia de las especies en este camino unidireccional. También, este mito de la colonialidad establece como humanos superiores a los hombres, quienes hacen la cultura mientras que las mujeres, como humanos inferiores, sólo la transmiten. ${ }^{24}$

Para la sociedad de Puerto Madryn, la cara visible de la comunidad Pu Fotum Mapu es su lonko (Cacique) Angel Ñanco, de origen tehuelche y a pesar de que la comunidad se denomina "mapuche tehuelche", pocos conocen que existen miembros con ascendencia mapuche (de hecho, para muchos originarios, hoy por hoy no se trata de dos pueblos distintos). ${ }^{25}$

Yo le dije a mi hijo, le digo pero ellos no le dan importancia a lo mío [...] ellos palparon mucho lo del padre, la historia del padre, quién es su papá, de dónde vino, quién es, todo, ¿no? Y a veces, cuando les toca hablar de mí, ino?, yo me siento mal ¿Por qué me niegan? ¿Por qué? ¿Por qué tienen esa descarez de negarme? Es como que no asumen que yo soy descendiente de mapuche.

E: $Y$ vos ¿qué pensás? ¿A qué responde eso?

G: No, es porque a ellos es cómo los criamos, como en eso de que su papá era el descendiente, que su papá era el originario.

Sin embargo, gran parte de la reproducción de la cultura mapuche pasa por manos de Gladys Villar Ñanco y se expresa en la creación de un territorio comunitario propio. Ella viste el trariwe ${ }^{27}$ y la trapelacucha ${ }^{28}$ para las ceremonias, narra versiones modificadas de

${ }^{23}$ LUGONES, 2008, p. 87, Las comillas son de la autora.

24 "La cultura moldea nuestras creencias. Percibimos la versión de la realidad que ella comunica. Paradigmas dominantes, conceptos predefinidos que existen como incuestionables, imposibles de desafiar, nos son transmitidos a través de la cultura. La cultura la hacen aquellos en el poder -hombres. Los varones hacen las reglas y las leyes; las mujeres las transmiten" (ANZALDÚA, 2004).

${ }^{25}$ Tema de un debate aún pendiente.

${ }^{26}$ Entrevista a Gladys Villar Ñanco, enero 2011.

${ }^{27}$ Cinturón tejido por ellas mismas que sujeta el küpam (vestido).

${ }^{28}$ Principal conjunto ornamental que no sólo engala sino especifica identidad. Se trata de una alhaja pectoral que cae sobre el pecho femenino, cuyo diseño muestra un águila de dos cabezas en el ápice; bajo las aves cuelgan dos pillanes (fuerzas extraordinarias y poderosas) y luego un conjunto de cadenas que culminan casi siempre en una placa de la cual cuelgan monedas o campanas. 
las/los Sumpall ${ }^{29}$ en las escuelas pero sobre todo mantiene vivo el territorio de la Comunidad Mapuhe Tehuelche Pu Fotum Mapu. Allí, levantó (dirigió) dos winoytripantu ${ }^{30}$ (año que vuelve) y otras ceremonias de menor importancia junto a su hija mayor, además de realizar talleres, campamentos y huerta.

Llevada al extremo, la posición esencialista sobre la relación Mujer-Naturaleza llevaría a pensar que no existe opresión entre mujeres ni patriarcado entre culturas originarias, por encontrarse en un supuesto estadio anterior, alejadas de la modernidad occidental. Sin embargo, la militante y werkén (vocera ) Moira Millán nos recuerda que

La penetración colonizadora a nuestro pueblo ha generado machismo en nuestras comunidades, y la mujer mapuche se ve hoy luchando por recuperar identidad, dignidad y respeto ya no solo ante la sociedad blanca europeísta sino también ante sus propios hombres [...] La violencia doméstica y conyugal lamentablemente es un problema que aún no se ha conflictivizado desde las organizaciones mapuches en general, recuerdo hace unos cuatro años atrás haber viajado al gulumapu (Chile) para solidarizarme con los presos políticos, al visitar la cárcel de Angol me enteré del caso de un lamngen preso cuya esposa lo había abandonado posteriormente a su arresto para juntarse con el carabinero que lo detuvo, ese hecho me sorprendió mucho y las organizaciones veían muy mal la actitud de la esposa, luego tuve la posibilidad de entrevistarme con ella y me relató el mal trato que recibía por parte de su marido y la violencia de la que era víctima, comprendí que para ella la lucha mapuche por la libertad no era más que seguir reproduciendo la esclavitud. ${ }^{31}$

En este sentido, Paula Castia recuerda el proceso de organización del Primer Encuentro de Mujeres en Puerto Madryn con mucho dolor, porque terminó quedando en manos de los hombres de la comunidad. El lonko no permitió que las mujeres firmen los comunicados de difusión, ni que los participantes sean exclusivamente mujeres.

El problema es el machismo de las mujeres... Pensamos un encuentro de mujeres, hecho por mujeres, organizado por mujeres. Y no lo vimos nunca como algo feminista eso. Pensar algo para las mujeres, hecho por mujeres. $Y$ los hombres a eso lo ven como feminismo. No lo ven como feminismo que defiende los derechos de las mujeres. Lo ven como un feminismo-machismo, a ver si me entendés. Como diciendo discriminamos a los hombres, no queremos a los hombres. $Y$ no se trataba de eso. ${ }^{32}$

Visualizar la dominación de género hoy en las comunidades originarias, no significa dar por sentado de que siempre fue así. Las teóricas feministas citadas en este apartado, dan cuenta construcción diferencial del género en términos raciales.

Es importante entender hasta qué punto la imposición de este sistema de género fue tanto constitutiva de la colonialidad del poder como la colonialidad del poder fue constitutiva de este sistema de género. La relación entre ellos sigue una lógica de constitución mutua [...] Problematizar el dimorfismo biológico y considerar la relación entre el dimorfismo biológico y la construcción dicotómica de género es central para

\footnotetext{
${ }^{29}$ Relato íntimamente relacionados con lo femenino.

${ }^{30}$ Así se denomina al año nuevo mapuche cuando comienza el equinoccio de invierno, el 21 de junio aproximadamente, que corresponde al día más corto del año a partir del cual todo comienza a renacer marcando el inicio de un nuevo ciclo.

${ }^{31}$ Moira MILLAN, 2011, p. 134-135. En una reciente entrevista a Segato, Karina Bidaseca resalta que "Ias afinidades semánticas entre cuerpo y territorio, dentro del paradigma colonial, son infinitas... Posiblemente el cuerpo indio no tenga, desde una perspectiva pre-colonial o no-colonial, esos mismos significados. Pero la colonialidad se los asigna." Karina BIDASECA, 2014

${ }^{32}$ Entrevista a Paula Castia, marzo de 2012.
} 
entender el alcance, la profundidad, y las características del sistema de género colonial/ moderno. La reducción del género a lo privado, al control sobre el sexo y sus recursos y productos es una cuestión ideológica presentada ideológicamente como biológica, parte de la producción cognitiva de la modernidad que ha conceptualizado la raza como 'engenerizada' y al género como racializado de maneras particularmente diferenciadas entre los europeos-as/blancos-as y las gentes colonizadas/no-blancas. La raza no es ni más mítica ni más ficticia que el género -ambos son ficciones poderosas. ${ }^{33}$

Volviendo al primer subtítulo de este ensayo, mi planteo central postula que es necesario reconocer una relación de proximidad mujer- naturaleza para construir un pensamiento-acción emancipatorixs, porque cuestiona el carácter escindido y alienado en que la dominación mantiene nuestras vidas. Pero de ningún modo este acercamiento o comunicación con el ambiente puede resultar en un reforzamiento de la dicotomía colonialista naturaleza/cultura, mujer/hombre, inferior/superior. Ése es el riesgo que se corre cuando se esencializan los conceptos y se naturalizan las prácticas.

\section{Bibliografía}

ARGUINAGA, Margarita LANG, Miriam; MOKRANI, Dunia; SANTILLANA, Alejandra. "Pensar desde el feminismo: críticas y alternativas al desarrollo". In: Grupo Permanente de Trabajo sobre Alternativas al Desarrollo, Más allá del desarrollo. Bs. As., América Libre, 2012. p. 55-82.

BIDASECA, Karina. "Mujer y cuerpo bajo control". Revista Ñ, Diario Clarín, 8 fev. 2014. Entrevista a Rita Segato. Disponible en: <http://www.revistaenie.clarin.com/ideas/Rita-SegatoMujer-cuerpo-control_0_1081091894.html>. Acceso en: 10 abr. 2014.

LAMAS, Marta. "La antropología feminista y la categoría de género". Revista Nueva Antropología, México, v. 8, n. 30, p. 173-198, nov. 1986.

LUGONES, María. "Colonialidad y género", Tabula Rasa, Universidad Colegio Mayor de Cundinamarca, Bogotá, n. 9, p. 73-101, jul./dic. 2008.

MILLÁN, Moira. "Mujer mapuche. Explotación colonial sobre el territorio corporal". In: BIDASECA, Karina (Co.comp.). Feminismos y poscolonialidad. Descolonizando el feminismo desde y en América Latina. Bs. As.: Ed. Godot, 2011. p. 291-306.

ORTNER, Sherry B. "Is female to male as nature is to culture?" In: ROSALDO, Michelle Z.; LAMPHERE, Louise (Comps.). Woman, culture, and society. Stanford: Stanford University Press, 1974.

PRONUNCIAMIENTO del feminismo comunitario en la conferencia de los pueblos sobre cambio climático". Tiquipaya, Cochabamba, Bolivia, abril de 2010. Disponible en: </http:// old.kaosenlared.net/noticia/pronunciamiento-feminismo-comunitario-latinoamericanoconferencia-pueb>. Acceso en: 15 fev. 2014.

QUIJANO, Aníbal. "Colonialidad del poder, eurocentrismo y América latina". In: LANDER, Edgardo (Comp.). Colonialidad del Saber, Eurocentrismo y Ciencias Sociales. CLACSO, Bs. As., 2000. p. 201-246.

SANTOS, Boaventura de Sousa. La caída del ángelus novas: ensayos para una nueva teoría social y una nueva práctica polític. Bogotá: Ediciones Antropos, 2003.

SEGATO, Rita. "Género y colonialidad: en busca de claves de lectura y de un vocabulario estratégico descolonial". In: BIDASECA, Karina (Coo-comp). Feminismos y poscolonialidad. Descolonizando el feminismo desde y en América Latina, Bs. As.: Ed. Godot, 201 1. p. 291306.

${ }^{33}$ LUGONES, 2008, p. 93-94

654 Estudos Feministas, Florianópolis, 22(2): 647-655, maio-agosto/2014 
STOLCKE, Verena. "¿Es el sexo para el género, lo que la raza para la etnicidad... y la naturaleza para la sociedad?" Revista Cultura y Política, Universidad Autónoma Metropolitana, DF, México, n. 14, p. 25-60, 2000.

WEINSTOCK, Ana Mariel. "Si a la vida, no a la mina". Voces y acciones confrontando el modelo de desarrollo en Patagonia. Alemania: Editorial Académica Española, 2012, p. 23-46.

[Recebido em fevereiro de 2014 e aceito para publicação em abril de 2014]

\section{Contributions of Feminism to Socioenvironmental Fight}

Abstract: It was from my Master's thesis that I became interested in the intersections between environmental thought and feminist theorizations. The Conference on pos and decolonial feminisms in Buenos Aires (2010 y 2012), organized by the program "Postcoloniality, border and crossborder thinking in feminist studies" (IDAES-UNSAM), provided an ideal setting for these discussions. This essay is a first attempt to address, from a feminist perspective, current socioenvironmental fights on land, territory and the model of development that take place in the Province of Chubut. Key Words: Woman; Nature; Oppression; Gender; Originary Worldview. 